\title{
A Novel Analysis Method of Pre-adjusting System for Self-interference Cancellation in Analog Domain
}

\author{
Hui-Hui $\mathrm{Li}^{1 *}$, Nan Chen ${ }^{1}$, and Xiandeng $\mathrm{He}^{1}$ \\ ${ }^{1}$ Skate Key Laboratory of Integrated Services Networks, XiDian University, Xi'an, Shaanxi, China
}

\begin{abstract}
Pre-adjusting system, which computes the attenuation coefficients of self-interference cancellation, is one of the most important parts in a practical self-interference cancellation system. In this paper, a new way is given to analyze the pre-adjusting system in analog domain, and a clear theoretical analysis is carried out. Meanwhile, a method for solving the optimal attenuation vector is introduced. The simulation results show that the Interference Cancellation Ratio(ICR) can reach up to $62.8 \mathrm{~dB}$ in an ideal environment and $50.4 \mathrm{~dB}$ in a real environment, which are much better than the ordinary demand of $40 \mathrm{~dB}$ in analog domain. Finally, an amplitude modulation signal is verified in our method, with shows good performance, too.
\end{abstract}

\section{Introduction}

With the rapid development of wireless communication technology, a large number of wireless electronic devices have been widely used in various fields[1]. The electromagnetic interference is being produced when people are enjoying the convenience of these equipments, especially in a limitted range of space, where a variety of radio transmission devices compete for a certain spectrum of resources[2]. These mutual interferences among the radio transmission equipments have seriously affected their functions. Interference cancellation is becoming important in both advanced wireless devices and future wireless networks[3]. In limitted space of communication platforms, the radios are often selfinterference limited rather than external-interference or noise-limited. Meanwhile, in the fifth generation of wireless mobile communications, the co-time co-frequency full duplex(CCFD) technology will play an important role[1]. What's more, the self-interference cancellation problem is the core challenge of CCFD. So it's meaningful to do some research on self-interference cancellation.

Certainly, self-interference cancellation can be divided into three stages: antenna cancellation, analog domain cancellation, and digital domain cancellation[2][4]. In this paper, we major in the analog domain cancellation. Earlier studies on the self-interference cancellation algorithms in the analog domain mainly focus on the structure. The authors in [4]-[6] provided a self-interference estimation structure in the analog domain, and adjusted

\footnotetext{
*Corresponding author: 15891027098@163.com
} 
its phase, amplitude and delay to cancel the self-interference. Besides, [7]-[11] gave some skills to got the attenuation coefficients. In this paper, the algorithm is optimized and verified in the actual system.

This paper studies the interference cancellation based on a Six-channels Interference Canceller as Fig 1, which is made by our laboratory. In Fig 1, every channel means a different frequency, widely from $30 \mathrm{MHz}$ to $512 \mathrm{MHz}$ randomly. Each channel is similar to others. And details of the single channel is given as Fig 2, which will be described below. Actually the canceller is divided into two parts from a functional point of view. One is preadjusting system and the other is cancellation system. The pre-adjusting system which estimate the attenuation coefficients, and depends on them, the cancellation system do the cancellation job. Naturally, the pre-adjusting system is the pivotal one. In this paper, a new analysis method based on mathematical equation is proved. Firstly, a structure of preadjusting system is given. Secondly, some theoretical analyses are done in detail. Thirdly, the method is verified by System View.

This paper is structured as follows. Section 2 presents the model of the pre-adjusting system. At the same time, a theoretical analysis is given in detail. Simulation results are given in Section 3. Conclusions are made in Section 4.

\section{System model and analysis}

In our Six-channels Interference Canceller, each channel is similar to others. Without loss of generality, as show in Fig 2, a general pre-adjusting system model of single channel is given.

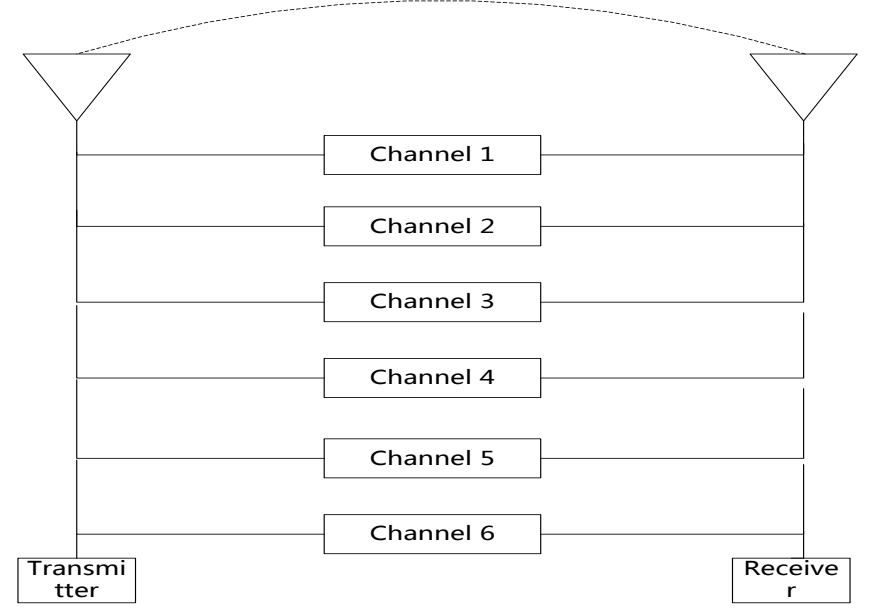

Fig. 1. Six-channels interference canceller

Obviously, the block of "Transmitter" transmits interference signal and the block of "Receive Signal" receives signals. There must be something to explain that the desired signal is not given in this structure. Because the canceller has three steps to finish a job: first, the pre-adjusting system finds the attenuation coefficients; second, the cancellation system do the cancellation job by using the attenuation coefficients from the first step; third, the desired signal can be transmitted.

In Fig 2, the block of "Reference Signal" get some portion of the signal from the block "Transmitter". Then we get $S_{1}(t)$ and $S_{2}(t)$ after the block of "Hibert Transform". The value of the block "Control" and "Integrals" is to get attenuation coefficients. Finally, the block "Merger Offset" merges signals. 


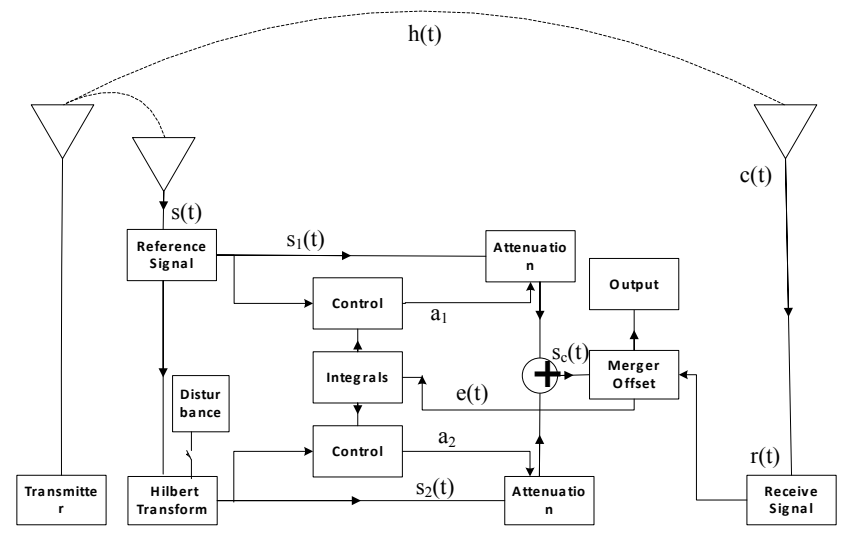

Fig .2. Pre-adjusting system of single channel

\subsection{Theoretical analysis}

As show in Fig 2, $s(t)$ is the reference signal which is coupled from transmitting antenna. The channel characteristics of transmission path is $h(t)$.

$$
h(t)=a \delta(t-\tau) e^{j \theta}
$$

Where $a, \tau$ are the amplitude attenuation and time delay of the self-interference channel, respectively. And $\theta$ is the random phase shift of this channel. Then $s_{1}(t)$ and $s_{2}(t)$ are the same phase and orthogonal component of $s(t)$,respectively. The orthogonal component is achieved by Hibert filter. So the received signal can be written as

$$
\begin{aligned}
r(t) & =c(t)+n(t)=\operatorname{Re}\{[s(t)+j \hat{s}(t)] * h(t)\}+n(t) \\
& =\operatorname{Re}\left\{\int_{-\infty}^{+\infty} h(\tau-t)[s(\tau)+j \hat{s}(\tau)] d \tau\right\}+n(t) \\
& =\operatorname{Re}\left\{a[s(t-\tau)+j \hat{s}(t-\tau)] e^{j \theta}\right\}+n(t)
\end{aligned}
$$

where $c(t)$ is the real self-interference and $n(t)$ represents the additive white Gaussian noise. And $\mathrm{r}(\mathrm{t})$ is a receive signal which contains interference signal and noise. Meanwhile, * represents the convolution operation. $\operatorname{Re}\{x\}$ denotes the real part of $x$, and $\hat{s}(t-\tau)$ is the Hibert transform of $s(t-\tau)$.

Given $\vec{a}=\left[a_{1}, a_{2}\right]^{T}$, where $a_{1}$ and $a_{2}$ are the attenuation coefficients of the same phase and orthogonal branch. So it is supposed that $\vec{s}(t)=\left[s_{1}(t), s_{2}(t)\right]^{T}$. Obviously, from Fig 1, the equivalent cancellation signal, by orthogonal vector synthesized, can be given as

$$
s_{\mathrm{c}}(t)=\vec{a}^{T} \cdot \vec{s}(t)
$$


where $s_{\mathrm{c}}(t)$ is the synthetic signal of the same phase and the orthogonal component. It's easy to see that the phase and amplitude of the synthetic signal can be adjusted by changing the elements of $\vec{a}$. From (3) and Fig 2, the residual self-interference signal after offsetting is written as

$$
e(t)=r(t)-s_{c}(t)=r(t)-\vec{a}^{T} \cdot \vec{s}(t)
$$

According to the irrelevance between $c(t)$ and $n(t)$, the analysis of the residual selfinterference signal is equivalent to calculate the optimal attenuation coefficient vector $\vec{a}$. The aim is to minimize the power of the residual self-interference signal, just as

$$
\min _{\vec{a}} E\left\{|e(t)|^{2}\right\}=\min _{\vec{a}} E\left\{(\mathrm{r}(t)-\vec{a} \bullet \vec{s}(t))^{2}\right\}
$$

\subsection{Optimal attenuation coefficients solution}

From the previous section, the problem of self-interference cancellation can be changed to get the optimal solution of $\vec{a}$ when the power of residual signal is minimum. Considering the details of the error signal,

$$
\begin{aligned}
& E\left\{|e(t)|^{2}\right\}=E\left\{\left[r(t)-\vec{a}^{T} \cdot \vec{s}(t)\right]\left[r(t)-\vec{a}^{T} \cdot \vec{s}(t)\right]\right\} \\
& =E\left\{r(t)^{2}-2 r(t) \vec{a}^{T} \cdot \vec{s}(t)+\vec{a}^{T} \vec{s}(t) \bullet \vec{s}^{T} \vec{a}\right\}
\end{aligned}
$$

It can be assumed that the power of self-interference signal is $P_{C}$ and the power of noise is $\sigma_{n}{ }^{2}$. Then, from (2) and (6), a simplified form of (6) can be expressed as

$$
\begin{aligned}
E\left\{|e(t)|^{2}\right\} & =E\left\{c(t)^{2}+n(t)^{2}-2[c(t)+n(t)] \vec{a}^{T} \cdot \vec{s}(t)+\vec{a}^{T} \vec{s}(t) \bullet \vec{s}^{T}(t) \vec{a}\right\} \\
& =P_{C}+\sigma_{n}{ }^{2}-2 E\left\{c(t) \vec{a}^{T} \vec{s}(t)\right\}+E\left\{\vec{a}^{T} \vec{s}(t) \cdot \vec{s}^{T}(t) \vec{a}\right\}
\end{aligned}
$$

Without loss of generality, suppose self-interference signal and reference signal are all stable. Then,

$$
\begin{aligned}
\vec{p} & =E\{c(t) \vec{s}(t)\} \\
\vec{R} & =E\left\{\vec{s}(t) \vec{s}^{T}(t)\right\}
\end{aligned}
$$

where $\vec{p}$ is the correlation vector between reference signal and received signal, and $\vec{R}$ is the autocorrelation matrix of the reference signal. then (7) can be simplified as

$$
E\left\{|e(t)|^{2}\right\}=P_{C}+\sigma_{n}{ }^{2}-2 \vec{a}^{T} \vec{p}+\vec{a}^{T} \vec{R} \vec{a}
$$

From (5), the optimal solution can be get as by $\vec{a}$ when (10) gets the minimum. That is $\vec{a}$ should satisfy the requirement of (11). 


$$
\frac{\partial E\left\{|e(t)|^{2}\right\}}{\partial \vec{a}}=-2 \vec{p}+2 \vec{R} \vec{a}=0
$$

It can be seen that the error mean square function is a quadratic function of the attenuation coefficient vector $\vec{a}$. To solving the optimal value of the attenuation coefficient is to get the result of the Wiener Hoff's equation defined by (11).

However, the accurate estimation of the correlation vector $\vec{p}$ and the autocorrelation matrix $\vec{R}$ are difficult to get. However, the recursive method of the conventional Wiener Hoff's equation is restricted by the range of the eigenvalue of the relevant matrix , and it is impossible to get a fast and accurate answer[12]. In addition, if the relevant matrix from (11) is a singular matrix, the optimal solution can't be get usually. Therefore, we propose a method of iterative weighting to estimate the correlation vector $\vec{p}$ and the autocorrelation matrix $\vec{R}$, effectively. Furthermore, the least squares solution is obtained by using the generalized inverse matrix of autocorrelation matrix from (11). Then, the procedure can be expressed as (12), (13), (14) and (15).

$$
\begin{gathered}
\vec{p}_{n}=\mu \vec{p}_{n-1}+(1-\mu) \vec{p}^{\prime} \\
\vec{R}_{n}=\mu \vec{R}_{n-1}+(1-\mu) \vec{R}^{\prime} \\
\vec{p}^{\prime}=\int_{t_{0}^{1}}^{t_{1}} r(t) \vec{s}(t) d t \\
\vec{R}^{\prime}=\int_{t_{0}}^{t_{1}} \vec{s}(t) \vec{s}^{T}(t) d t
\end{gathered}
$$

where $\mu$ is the weighting factor, $t_{0}$ and $t_{1}$ are the start time and end time, respectively. $\vec{p}_{n}$ is the nth iteration estimates of the correlation vector and $\vec{R}_{n}$ is the autocorrelation matrix. The initial values $\vec{p}_{0}$ and $\vec{R}_{0}$ can be set to zero.

On the basis of (11) to (15), the optimal value of the attenuation coefficient vector, corresponding to the nth iteration, is

$$
\vec{a}_{n}=\vec{R}_{n}^{+} \vec{p}_{n}
$$


where $\vec{R}_{n}^{+}$is the Moore-Penrose inverse of the autocorrelation matrix ${ }_{n}{ }_{n}$. When ${ }_{n}{ }_{n}$ is not singular, $\vec{R}_{n}^{+}=\vec{R}_{n}^{-}$.

\subsection{Interference cancellation ratio (ICR)}

In addition to the convergence rate, another important performance indicator of preadjusting system is the ICR. According to section B, we can give a define of ICR as (17).

$$
I C R=10 \lg \left(\frac{E\left\{|c(t)|^{2}\right\}}{E\left\{|e(t)|^{2}\right\}-E\left\{|n(t)|^{2}\right\}}\right)=10 \lg \left(\frac{P_{C}}{E\left\{|e(t)|^{2}\right\}-\sigma_{n}^{2}}\right)
$$

Then, considering all the condition, the simplest form of ICR can be achieved as (18).

$$
I C R=20 \lg \left(\frac{A_{c}}{A_{e}}\right)
$$

where $A_{c}$ is the amplitude of self-interference and $A_{e}$ is the amplitude of the residual signal.

\section{Simulation results}

To verify the self-interference cancellation performance of the pre-adjusting system model as show in Fig 2, simulations are performed by the System View simulation software in this section. And some analysis also are done in an ideal environment and a real environment, respectively.

Without loss of generality, the reference signal is a sinusoidal signal . Its frequency is $300 \mathrm{MHz}$. At the same time, the number of samples in this paper is set to 300,000 in order to have better statistical properties.

As the reference signal may have other complex representations, we consider an amplitude modulation signal as reference signal, and other parameters remain the same.

\subsection{Ideal environment}

In this section, the disturbance is not given. Fig 3 shows the amplititude of the residual selfinterference signal. Fig 4 and Fig 5 displays the frequency spectrum characteristics of the self-interference signal before and after cancellation. It is clear that the method of this paper has a significant effect on self-interference cancellation.

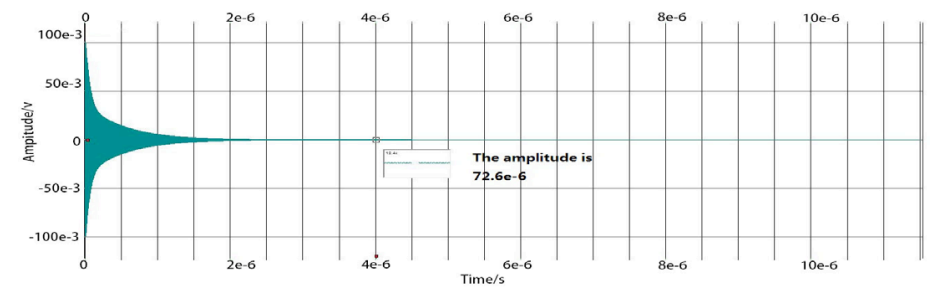

Fig. 3. Residual signal of self-interference in an ideal environment. 


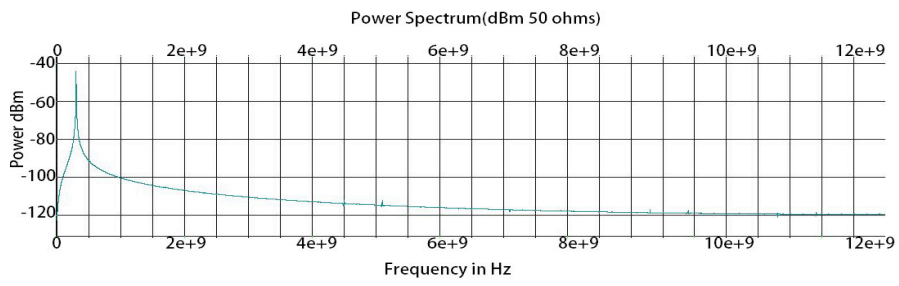

Fig. 4. The frequency spectrum of residual signal in an ideal environment.

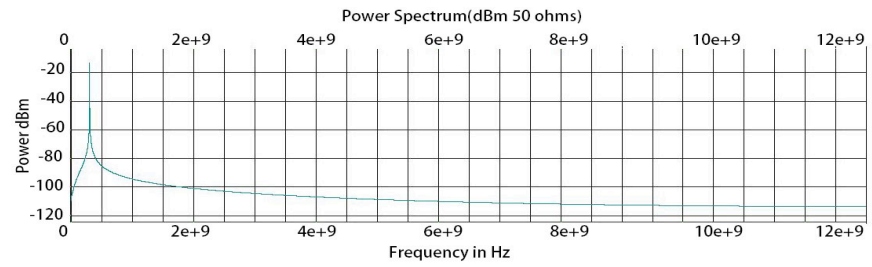

Fig. 5. The frequency spectrum of self-interference before cancellation

$$
I C R=20 \lg \left(\frac{A_{c}}{A_{e}}\right)=20 \lg \left(\frac{0.1}{72.6 \times 10^{-6}}\right)=62.8 \mathrm{~dB}
$$

As show in Fig 4, it is only $2 \mu \mathrm{s}$ when the system converges to a steady state. Actually, the convergence process is to find the optimal attenuation vector. When the waveform tends to be stable, the optimal solution is found which is promised by 300,000 samples. Then, the ICR is $62.8 \mathrm{~dB}$, which is shown in Fig and calculated by (19).

\subsection{Real environment}

As it is difficult for us to get an ideal Hilbert filter in reality, the inherent characteristic should be considered. In the simulation, we simulate the non-ideal part by give a disturbance in $-60 \mathrm{dBm}$. Before interference cancellation, the mixed frequency spectrum of the self-interference and the disturbance is given by Fig 8 .

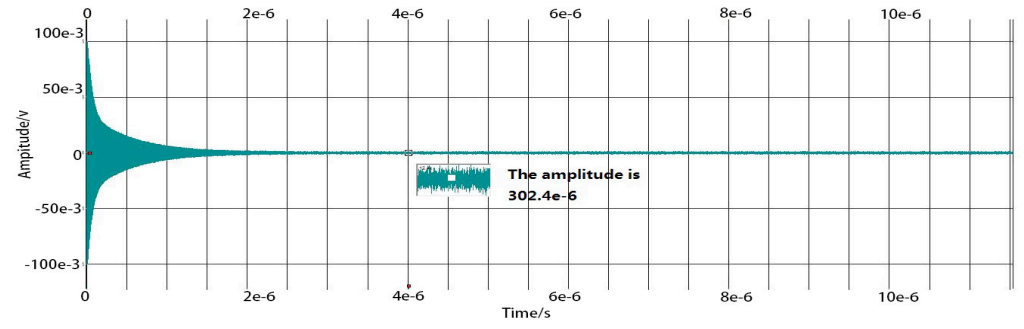

Fig. 6. Residual signal of self-interference in a real environment.

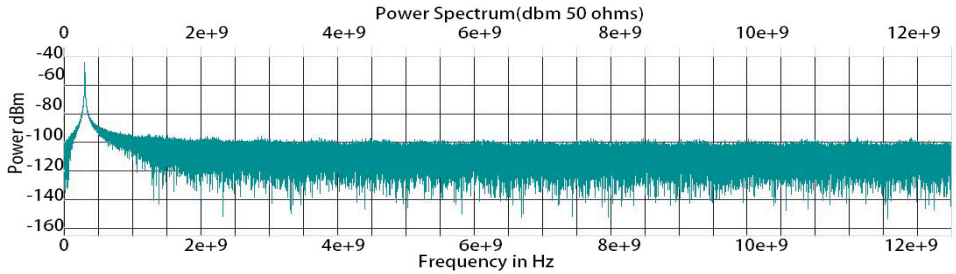

Fig. 7. The frequency spectrum of residual signal in a real environment. 


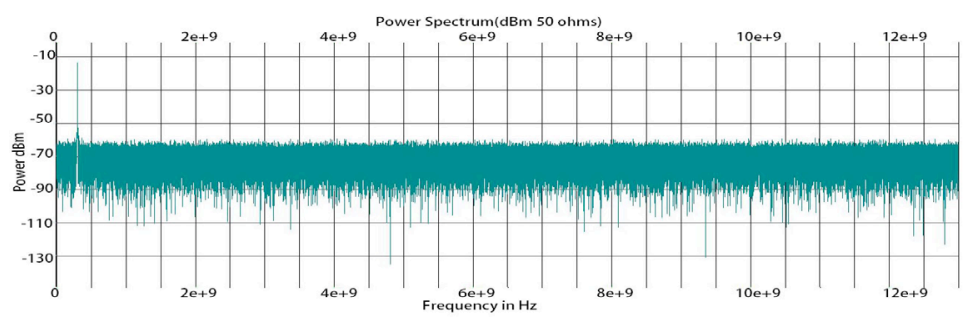

Fig. 8. The frequency spectrum in a real environment before interference cancellation.

Comparing the ordinates in Fig 7 and Fig 8, it can be seen that the amplitude has a disdinct decline after interference cancellation.

In Fig 6, it can be observed that the convergence time is about $2.5 \mu$ s which means we will get an optimal solution after $2.5 \mu \mathrm{s}$. The ICR is $50.4 \mathrm{~dB}$ which is shown in Fig 6 and calculated by (20), and the disturbance decrease $40 \mathrm{dBm}$, which is shown in Fig 7 and calculated by (21).

$$
\begin{gathered}
I C R=20 \lg \left(\frac{A_{c}}{A_{e}}\right)=20 \lg \left(\frac{0.1}{302.4 \times 10^{-6}}\right)=50.4 d B \\
-60 d B m-(-100 d B m)=40 d B
\end{gathered}
$$

Comparing Fig 4 with Fig 7, it can be seen that the main frequency components at $300 \mathrm{MHz}$ are close to each other, about $-45 \mathrm{dBm}$. Note that when take the disturbance in, our method is influenced. It is the inherent characteristic of Hilbert filter that makes the self-interference cancellation performance be deteriorated. Obviously, this performance is $10.4 \mathrm{~dB}$ better than the ordinary demand of $40 \mathrm{~dB}$ in analog domain[13].

\subsection{Amplitude modulated signal}

In reality, there are lots of modulated signals. In this paper, the amplitude modulated signal is considered. Now, the reference signal can be expressed as (22),

$$
s(t)=A \cos (2 \pi f t) \cos \left(2 \pi f_{c} t\right)
$$

where $A$ is the amplitude, $f$ and $f_{c}$ are the frequency of signal and carrier, respectively. The values of $f, f_{c}$ are $300 \mathrm{MHz}$ and $3 \mathrm{GHz}$ respectively. It is possible to get (23) by simplifying (22).

$$
s(t)=\frac{A}{2}\left\{\cos 2 \pi\left(f_{c}+f\right) t+\cos 2 \pi\left(f_{c}-f\right) t\right\}
$$

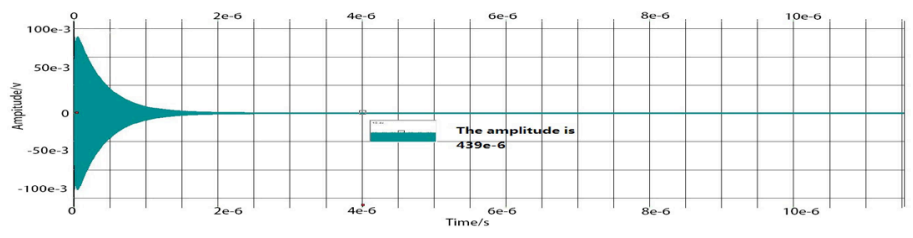

Fig. 9. Residual signal of self-interference. 


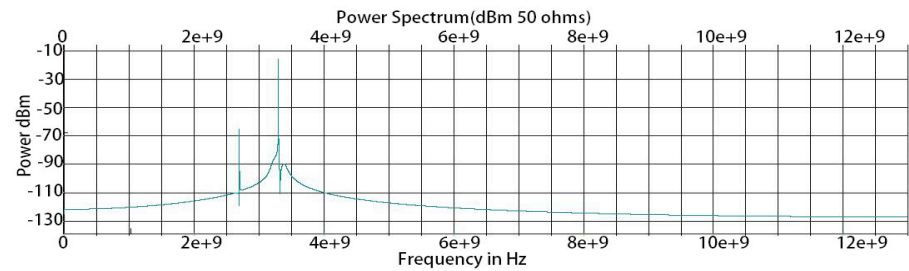

Fig. 10. The frequency spectrum before interference cancellation.

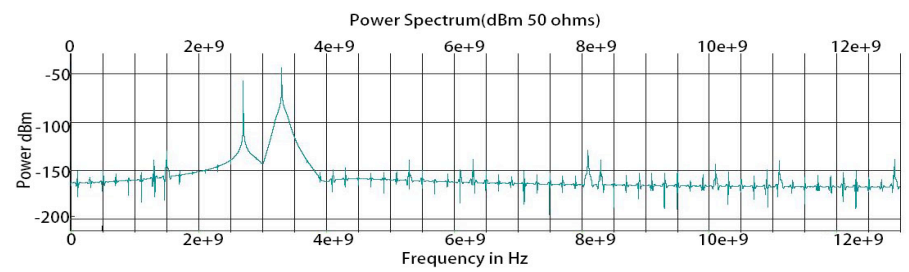

Fig. 11. The frequency spectrum after interference cancellation

$$
I C R=20 \lg \left(\frac{A_{c}}{A_{e}}\right)=20 \lg \left(\frac{0.05}{439 \times 10^{-6}}\right)=41.1 \mathrm{~dB}
$$

Depending on the symmetry, only the upper sideband need to be considered. The simulation of residual signal is expressed as Fig 9. Comparing Fig 10 with Fig 11, we can see that our method has a good performance in $3.3 \mathrm{GHz}$. Meanwhile, there is a spectrum line in $2.7 \mathrm{GHz}$, which is produced by the non-ideal characteristics of the band-pass filter. And the ICR is $41.1 \mathrm{~dB}$ from (24), which has an advantage of $1.1 \mathrm{~dB}$ than [13]. Obviously, it has a good job.

\section{Conclusion}

Based on the Six-channels Interference Canceller, this paper presents a new method of preadjusting system. Not only the clearly theoretical analysis is proposed, but also a way to solve the optimal attenuation vector is given. The results of simulation in System View tell us that this method has a $10.4 \mathrm{~dB}$ better performance than the ordinary demand. Meanwhile, it also has a good performance when the reference signal has a complex form. At the same time, a large number of samples are used in this paper to achieve a better performance, which increases the time loss. Therefore, the algorithm would be optimized in the future. Meanwhile, the environment of CCFD will be studied at a later time.

\section{References}

1. J. Lu, ZTE TECNOLOGY JOURNAL.23,2(2017)

2. J. Wang, H. Zhao, Y. Tang, IEEE ICC, pp. 5622-5627(2016)

3. H. Wang, J. Wang, H. Li, Jou\&Mag, 23,858(2012)

4. J. Choi, M. Jain, K. Srinivasan, P. Levis, S. Katti , MOBICOM, 49,1(2010)

5. M. Jain, J. Choi, T. Kim, D. Bharadia, S. Seth, ICMCN. ACM pp. 301-312(2011)

6. S. Hong, J. Mehlman, S. Katti, ASCCR, 42,37(2012)

7. X. Liu, J. Liu, F. Zhao, W. Zhang, IEEE ICCPS, PP. 138-141(2015)

8. J. Liu, H. Quan, Z. Li, D. Liu, H. Zhao, J. E\&I. T 39,654(2017)

9. I. Hwang, B. Song, N. Cong, SS. Soliman, IEEE Jou\&Mag, 34,2838(2016) 
10. Y. Xie, J. Liu, M. Li,S. Ouyang, G. Liao, Jou on Comm, 36,122(2015)

11. Y. Zhao, J. Lei, Ele Sci \& Tec, 35,145(2016)

12. S. HAYKAN, Adaptive Filter Theory, pp. 440-465(2008)

13. Y. Tang, Full Duplex Principles and Applications, pp. 34-173(2016) 